\title{
Frequency of coexistent carcinoma in sessile serrated adenoma/polyps and traditional serrated adenomas removed by endoscopic resection
}

Authors

Institutions
Hirotsugu Saiki ${ }^{1}$, Tsutomu Nishida ${ }^{1}$, Masashi Yamamoto ${ }^{1}$, Shiro Hayashi' ${ }^{1}$, Hiromi Shimakoshi ${ }^{1}$, Akiyoshi Shimoda ${ }^{1}$, Takahiro Amano ${ }^{1}$, Aisa Sakamoto ${ }^{1}$, Yuriko Otake ${ }^{1}$, Aya Sugimoto ${ }^{1}$, Kei Takahashi ${ }^{1}$, Kaori Mukai ${ }^{1}$, Tokuhiro Matsubara', Sachiko Nakajima' ${ }^{1}$, Koji Fukui ${ }^{1}$, Masami Inada ${ }^{1}$, Katsumi Yamamoto ${ }^{1,2}$, Ryozo Tokuda $^{3}$, Shiro Adachi ${ }^{3}$

Institutions are listed at the end of article. submitted:

24. November 2015 accepted after revision: 8. February 2016

\section{Bibliography}

DOI http://dx.doi.org/

10.1055/s-0042-103239

Published online: 30.3.2016

Endoscopy International Open

2016; 04: E451-E458

(c) Georg Thieme Verlag KG

Stuttgart · New York

E-ISSN 2196-9736

\section{Corresponding author}

\section{Tsutomu Nishida, MD}

Department of

Gastroenterology

Toyonaka Municipal Hospital

4-14-1 Shibahara, Toyonaka

Osaka 560-8565

Japan

Fax: +81-6-6858-3531

tnishida.gastro@gmail.com
Background and study aims: Sessile serrated adenoma/polyps (SSA/Ps) have a different potential than traditional adenomatous polyps for developing into malignant colorectal cancer. However, little is known about the coexistent cancer rate. Here, we evaluate the frequency of carcinoma in serrated polyps removed by endoscopic resection (ER).

Patients and methods: This was a retrospective single-center cohort study of consecutive patients with colorectal polyps who underwent ER from March 2003 to October 2014. We determined the frequency of serrated polyps among all resected colorectal polyps and analyzed the clinicopathological findings as well as the frequency and characteristics of coexistent carcinoma in the serrated polyps resected by ER based on pathology reports.

Results: A total of 21,048 polyps from 15,326 patients were identified, including 15,984 traditional adenomatous polyps (75.9\%), 621 SSA/Ps (3.0\%),

\section{Introduction \\ $\nabla$}

Colorectal polyps are histologically classified as neoplastic or nonneoplastic. Most colorectal cancers (CRCs) arise from neoplastic adenomatous polyps, and serrated polyps have recently been shown to have a different potential than traditional adenomatous polyps to develop into malignant CRC [ 1 - 3]. Serrated polyps are pathologically classified into three groups by the World Health Organization: hyperplastic polyps (HPs), sessile serrated adenoma/polyps (SSA/Ps), and traditional serrated adenomas (TSAs). Of these three types, SSA/Ps are considered the main premalignant lesions in the serrated neoplastic pathway to CRC, which represents 20 to 30 percent of all sporadic CRC cases [4]. SSA/Ps generally lack classic dysplasia. Foci of cytological dysplasia in SSA/Ps should be noted by the pathologist as a SSA/P with cytological dysplasia. These lesions are thought to be
136 traditional serrated adenomas (TSAs) (0.6\%), 1,121 hyperplastic polyps ( $5.3 \%$ ), and 3,186 polyps of other types (15.1\%). The clinical and endoscopic findings of SSA/Ps revealed a male predominance (68.6\%), with $61.7 \%$ of the polyps located in the proximal colon. Males accounted for $77.2 \%$ of all patients with TSAs, and $77.2 \%$ of these polyps were located in the distal colon. The mean sizes of the SSA/Ps and TSAs were 8.8 and $10.7 \mathrm{~mm}$, respectively. Among the SSA/Ps, $8(1.3 \%)$ cases had coexistent carcinoma, and $1(0.7 \%)$ patient with TSA showed coexistent carcinoma. In the patients with SSA/Ps, female sex and a tumor size $\geq 10 \mathrm{~mm}$ were predictive factors for coexistent carcinoma.

Conclusions: The frequency of SSA/Ps with carcinoma was lower than that for traditional adenoma. Female sex and tumor size $\geq 10 \mathrm{~mm}$ were significant predictive factors for coexistent carcinoma.

an important step toward carcinogenesis. In contrast, TSAs have diffuse but typically mild cytological dysplasia, and the classification systems for these polyps are still under development. Therefore, histological interpretation of these serrated polyp types differs among pathologists, which has led to a lack of knowledge regarding their true frequency.

Recently, it has been reported that polyps have the potential to develop into sporadic CRC via a series of molecular alterations, including BRAF mutation and $\mathrm{CpG}$ island methylation with epigenetic inactivation of the MLH1 mismatch repair gene, resulting in microsatellite instability (MSI) $[5,6]$. Many studies have suggested that TSAs and SSA/Ps have significant malignant potential and are associated with subsequent development of metachronous polyps [7-11].

Clinically, SSA/Ps are considered to be strongly associated with interval cancers after colonosco- 
py, which can originate from a rapidly progressing precursor lesion that evades detection. Failed attempts at endoscopic resection (ER) allow for the continued growth of these lesions. However, relatively little is known about the epidemiology of SSA/Ps and TSAs, particularly the coexistent cancer rates. This study was performed to determine the adenocarcinoma rates associated with SSA/Ps and TSAs based on the epidemiology of a large cohort. The rates were compared with those for other colorectal polyps resected during the same observation period. This report specifically describes the frequency of carcinoma in serrated polyps resected by ER.

\section{Patients and methods \\ $\nabla$}

This was a retrospective single-center cohort study of consecutive patients with colorectal polyps who underwent ER from March 2003 to October 2014 at Toyonaka Municipal Hospital. First, we evaluated the frequency of serrated polyps among all resected colorectal polyps. Next, we analyzed the clinicopathological findings, complete en bloc resection rate, and frequency and characteristics of coexistent carcinoma in serrated polyps resected by ER based on the pathology reports. This study was approved by the Institutional Review Board of Toyonaka Municipal Hospital.

\section{Indications for ER of colorectal polyps}

The typical indication for ER of a colorectal polyp is size $>5 \mathrm{~mm}$. However, the final decision to perform ER is made by each physician based on the patient's condition and bowel preparation. At our institution, diminutive polyps were allowed to be followed up without resection based on the judgment of each endoscopist. However, flat and depressed lesions that were difficult to distinguish from adenoma or carcinoma were generally resected. Patients who did not achieve a clean colon on initial colonoscopy underwent repeat ER to attain a clean colon, except in the case of diminutive polyps.

\section{Colonoscopy findings}

Locations of resected colorectal polyps were classified into two groups: "proximal," defined as the cecum, ascending colon, and transverse colon; and "distal," defined as the descending colon, sigmoid colon and rectum. Polyp size was endoscopically measured using standard clinical practices, such as by visual estimation, the open biopsy forceps method or use of a polypectomy snare. The macroscopic appearance of the polyp was classified according to the Paris endoscopic classification [12], and the data were annotated as follows: protruded or pedunculated (Ip) and intermediate or broad-based forms (semi-pedunculated [Isp], sessile [Is], and superficially elevated [IIa]). All colonoscopy findings were retrospectively collected from the endoscopy reports.

\section{Pathology findings}

Resected polyps were evaluated by experienced pathologists who worked full time at our hospital during the observation period. Basically, the histopathologic diagnosis was based on the morphologic features observed in hematoxylin- and eosinstained sections. SSA/Ps were diagnosed based on the following findings: crypt dilation, irregularly branching crypts, and horizontally arranged basal crypts (inverted T- and/or L-shaped crypts) [13]. TSAs were diagnosed based on the following find- ings: villiform growth, serrated epithelium associated with nuclear and cytological atypia, ectopic crypt formation, and eosinophilic cytoplasm. HPs were diagnosed based on findings of a serrated epithelial architecture in the upper part of the crypts, proliferative zones limited to the lower part of the crypts, and the absence of cytologic dysplasia. All data were collected from pathology reports.

\section{Statistical analysis}

All continuous variables are presented as the mean \pm standard deviation (SD), except for nonparametric variables, which are presented as the median and range. Categorical variables are presented as the number in each category or the frequency. Categorical variables were compared using the chi-squared test or Fisher's exact test, as appropriate. A receiver operating characteristic (ROC) curve analysis was used to determine the cutoff value for polyp size for coexisting adenocarcinoma. A value of $P<0.05$ was considered statistically significant. Statistical analyses were performed using JMP software (ver. 11.1.1, SAS Institute, Inc., Cary, NC).

\section{Results}

A total of 21,048 resected polyps from 15,326 patients $(70.0 \%$ men) were identified based on pathology reports from March 2003 to October 2014 at Toyonaka Municipal Hospital. There were 15,984 traditional adenomatous polyps (75.9\%; 95\% confidence interval [CI]: 75.4-76.5), 621 SSA/Ps (3.0\%; 95\% CI: $2.7-$ 3.2), 136 TSAs (0.6\%; 95\% CI: $0.6-0.8), 1122$ HPs (5.3\%; $95 \% \mathrm{CI}$ : $5.0-5.6)$, and 3,186 other polyps, including nonneoplastic lesions, inflammatory polyps, carcinoid tumors, lipomas, juvenile polyps and unclassified polyps (data not shown) ( Table 1 , - Fig. 1).

The clinical and endoscopic findings of SSA/Ps revealed a male sex $(68.6 \%)$ and sessile type (73.1\%) predominance, with $61.7 \%$ of the polyps located in the proximal colon ( Table 2$)$. The clinical and endoscopic findings of TSAs also revealed a male (77.2\%) and sessile type (65.4\%) predominance, with $77.2 \%$ of the polyps located in the distal colon ( Table 3 ). The mean sizes of the SSA/ Ps and TSAs were 8.8 and $10.7 \mathrm{~mm}$, respectively. The complete en

\begin{tabular}{|c|c|}
\hline Patient characteristics & $\mathbf{n}$ \\
\hline Total no. of patients & 15,326 \\
\hline Age, mean, years & $69 \pm 10$ \\
\hline Sex, male (\%) & $10,728,70.0 \%$ \\
\hline \multicolumn{2}{|l|}{ Lesion characteristics } \\
\hline Total no. of polyps & 21,048 \\
\hline $\begin{array}{l}\text { Traditional adenomatous polyps, n, \% } \\
\text { [95\% CI] }\end{array}$ & $15,984,75.9 \%[75.4-76.5]$ \\
\hline With adenocarcinoma, $\mathrm{N}, \%$ & $1,318,8.2 \%$ \\
\hline SSA/Ps, n, \% [95\% CI] & $621,3.0 \%[2.7-3.2]$ \\
\hline $\begin{array}{l}\text { With cytological atypia/adenocarcinoma, } \\
\mathrm{n}, \%\end{array}$ & $25 / 8,4.0 \% / 1.3 \%$ \\
\hline TSAs, n, \% [95\% Cl] & $136,0.7 \%[0.6-0.8]$ \\
\hline With adenocarcinoma, $\mathrm{n}, \%$ & $1,0.7 \%$ \\
\hline Hyperplastic polyps, n, \% [95\% Cl] & $1,121,5.3 \%[5.0-5.6]$ \\
\hline With adenocarcinoma, n, \% & $0,0 \%$ \\
\hline Other polyps, n, \% [95\% Cl] & $3,186,15.1 \%[14.7-15.6]$ \\
\hline
\end{tabular}

SSA/Ps: sessile serrated adenoma/polyps; TSAs: traditional serrated adenomas; HPs: hyperplastic polyps; Cl: confidence interval 

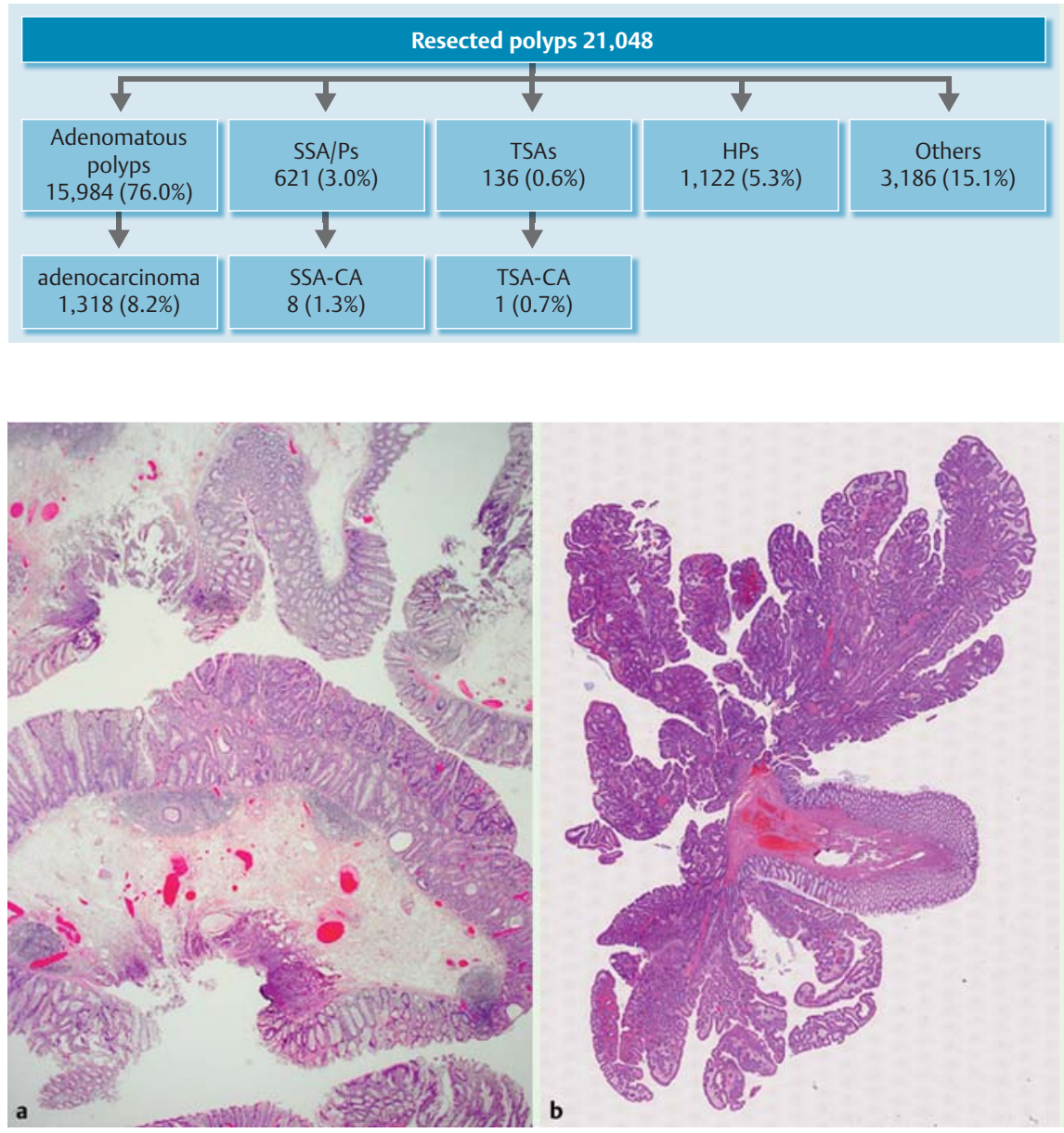

Fig. 1 Flowchart showing patients with resected colorectal polyps. SSA/Ps: sessile serrated adenoma/polyps; TSAs: traditional serrated adenomas; HPs: hyperplastic polyps; CA: carcinoma.

Fig. 2 Typical pathologic images of SSA/P (A) and TSA (B) with adenocarcinoma.

\begin{tabular}{|lll|}
\hline & SSA/Ps & With cytological dysplasia \\
\hline $\mathbf{n}$ & 621 & $25,4.0 \%$ \\
\hline $\begin{array}{l}\text { Sex, } \mathrm{n}(\%) \\
\text { Female }\end{array}$ & $426,68.6 \%$ & $20,80.0 \%$ \\
\hline Age, mean [range], years & $195,31.4 \%$ & $5,20.0 \%$ \\
\hline Location, $\mathrm{n}(\%)$ & $65.7(24-90)$ & $68.3(43-88)$ \\
Proximal (C/A/T) & & \\
Distal (D/S/R) & $62 / 180 / 141(383,61.7 \%)$ & $0 / 5 / 7(12,48.0 \%)$ \\
\hline Tumor size, mean (range), mm & $31 / 139 / 68(237,38.2 \%)$ & $3 / 7 / 3(13,52.0 \%)$ \\
\hline Macroscopic type & $8.8(3-33)$ & $9.8(5-33)$ \\
$0-$ Is & $387(62.3 \%)$ & $11(44.0 \%)$ \\
$0-$ Isp & $67(10.8 \%)$ & $6(24.0 \%)$ \\
$0-$ Ip & $12(1.9 \%)$ & $1(4.0 \%)$ \\
$0-$ Ila & $155(25.0 \%)$ & $7(28.0 \%)$ \\
\hline Complete en bloc resection & $352(65.2 \%)$ & $18(72.0 \%)$ \\
Cut end unclear or positive & $188(34.8 \%)$ & $7(28.0 \%)$ \\
Not described & 81 & 0 \\
\hline
\end{tabular}

Table 2 Characteristics of SSA/Ps.

SSA/Ps: sessile serrated adenoma/polyps; C: cecum; A: ascending colon; T: transverse colon; D: descending colon; S: sigmoid colon; R: rectum

bloc resection rates of SSA/Ps and TSAs were $65.2 \%$ and $62.4 \%$, respectively $(\bullet$ Table 2 and $\bullet$ Table 3 ).

Among the SSA/Ps, 25 (4.0\%) showed cytological atypia ( $\bullet$ Table 2). Of these, 8 had coexistent adenocarcinoma. Among the TSAs, 1 (0.7\%) showed coexistent adenocarcinoma ( $\bullet$ Table 4, $\bullet$ Fig. 1 , and $\odot$ Supplementary Table 1$). \odot$ Table 4 and $\odot$ Supplementary Table 1 provide the details of the SSA/Ps and TSAs with adenocarcinoma, and $\bullet$ Fig. 2 shows typical pathology images of SSA/Ps and TSAs with adenocarcinoma. Supplementary $\bullet$ Fig. 1 shows the endoscopic findings for each type of polyp. The optimal tumor size cut-off value for coexisting adenocarcinoma was 10 mm (AUC: 0.84) (๑ Fig. 3).

Adenocarcinoma was found in $8.2 \%$ of the traditional adenomatous polyps $(1,318 / 15,984)$ ( $\mathbf{~ F i g . ~} \mathbf{1})$. Therefore, the coexisting adenocarcinoma rate was significantly lower in SSA/Ps $(P<$ 0.0001 ) and TSAs (1 polyp; $P=0.0014$ ) than in traditional adenomatous polyps. $\odot$ Fig. 4 shows the distribution of the resected polyps according to size range. Most of the resected serrated 
Table 3 Characteristics of TSAs.

\begin{tabular}{|c|c|}
\hline & TSAs \\
\hline$n$ & 136 \\
\hline \multicolumn{2}{|l|}{ Sex, n (\%) } \\
\hline Male & $105(77.2 \%)$ \\
\hline Female & $31(22.8 \%)$ \\
\hline Age, mean (range), years & $66.7(35-92)$ \\
\hline \multicolumn{2}{|l|}{ Location, $\mathrm{n}(\%)$} \\
\hline Proximal $(C / A / T)$ & $2 / 10 / 19(31,22.8 \%)$ \\
\hline Distal (D/S/R) & $6 / 54 / 45(105,77.2 \%)$ \\
\hline Tumor size, mean (range), mm & $10.7(3-30)$ \\
\hline \multicolumn{2}{|l|}{ Macroscopic type } \\
\hline $0-$ Is & $29(21.3 \%)$ \\
\hline $0-$ Isp & $60(44.1 \%)$ \\
\hline $0-I p$ & $40(29.4 \%)$ \\
\hline 0 -Ila & $7(5.2 \%)$ \\
\hline Complete en bloc resection & $63(62.4 \%)$ \\
\hline Cut end unclear or positive & $38(37.6 \%)$ \\
\hline Not described & 35 \\
\hline
\end{tabular}

TSAs: traditional serrated adenomas; C: cecum; A: ascending colon; T: transverse colon; D: descending colon; S: sigmoid colon; R: rectum

Table 4 Serrated polyps with carcinoma.

\begin{tabular}{|lll|}
\hline & $\begin{array}{l}\text { SSA/Ps with } \\
\text { carcinoma }\end{array}$ & $\begin{array}{l}\text { TSAs with } \\
\text { carcinoma }\end{array}$ \\
\hline $\mathbf{n}$ & $8(1.3 \%)$ & $1(0.7 \%)$ \\
\hline $\begin{array}{l}\text { Sex, } \mathrm{n}, \% \\
\text { Male } \\
\text { Female }\end{array}$ & $2(20.0 \%)$ & $0(0 \%)$ \\
\hline Age, mean (range), years & $6(80.0 \%)$ & $1(100 \%)$ \\
\hline $\begin{array}{l}\text { Location, N, \% } \\
\text { C/A/T }\end{array}$ & $65.7(48-75)$ & 87 \\
D/S/R & $1 / 2 / 1(50.0 \%)$ & $0 / 0 / 0(0 \%)$ \\
\hline $\begin{array}{l}\text { Tumor size, mean (range), } \\
\text { mm }\end{array}$ & $0 / 3 / 1(50.0 \%)$ & $0 / 1 / 0(100 \%)$ \\
\hline Histological depth, M/SMM & $6 / 2$ & 10 \\
\hline $\begin{array}{l}\text { Macroscopic type } \\
0-\text { Is }\end{array}$ & $0(0 \%)$ & $1 / 0$ \\
$0-$ Isp & $4(50.0 \%)$ & $0(0 \%)$ \\
$0-$ Ip & $1(1.3 \%)$ & $1(100 \%)$ \\
$0-$ Ila & $3(37.5 \%)$ & $0(0 \%)$ \\
\hline $\begin{array}{l}\text { Complete en bloc } \\
\text { resection }\end{array}$ & $8(100 \%)$ & $1(100 \%)$ \\
\hline
\end{tabular}

SSA/Ps: sessile serrated adenoma/polyps; TSAs: traditional serrated adenomas; $\mathrm{C}$ : cecum; A: ascending colon; T: transverse colon; D: descending colon; S: sigmoid colon; R: rectum; M: mucosa; SM: submucosa.

polyps were between 6 and $20 \mathrm{~mm}$, while all the serrated polyps with adenocarcinoma were at least $10 \mathrm{~mm}$.

\section{Discussion}

Serrated lesions of the colorectum are histologically characterized by a serrated appearance of the crypt epithelium. A few decades ago, serrated lesions were termed "hyperplastic polyps" and were considered benign lesions [14]. Therefore, until recently, serrated polyps were not differentiated from hyperplastic polyps and were not thought to require polypectomy or surveillance. In the 1980 s, autopsy studies demonstrated that the percentage of Caucasian adults who had at least one serrated lesion ranged from $25 \%$ to $50 \%$ [ $15-18$ ]; these studies were conducted before specific serrated lesion subtypes were recognized. In 1990, the

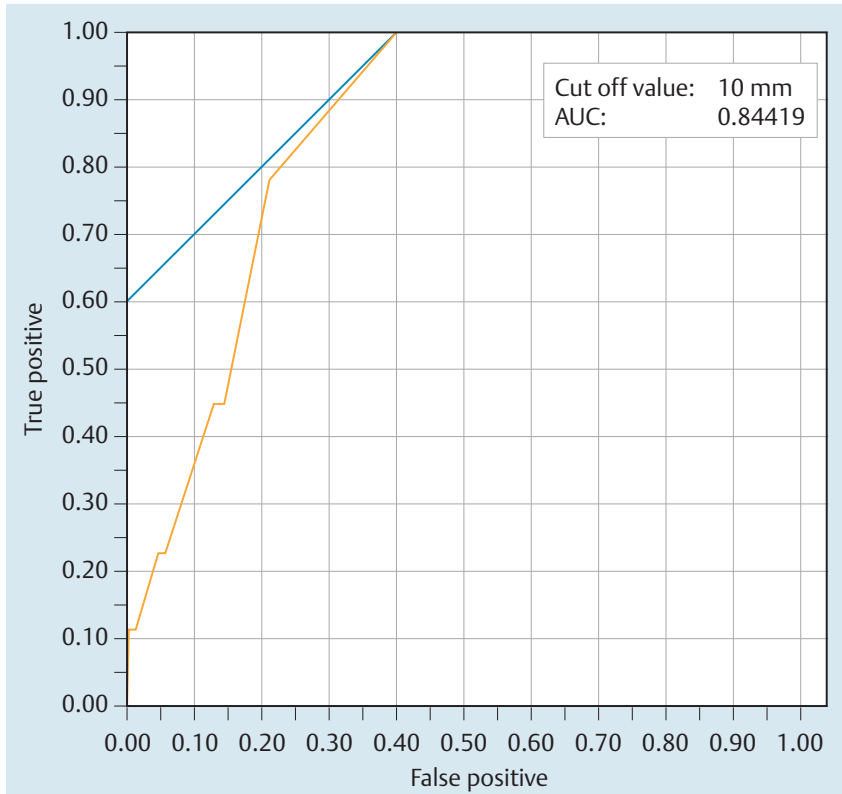

Fig. 3 Receiver operating characteristic (ROC) curves used to predict coexisting adenocarcinoma in patients with serrated polyps.

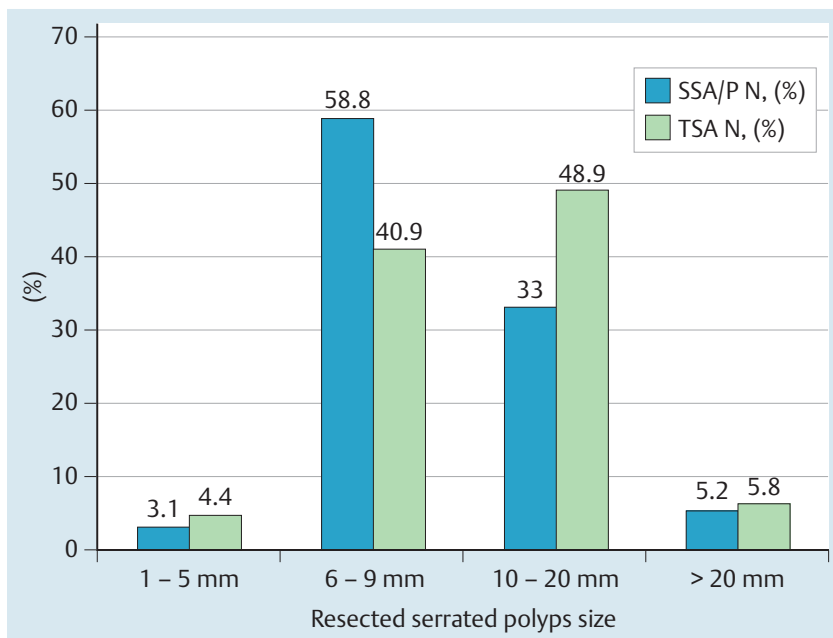

Fig.4 Distribution of serrated polyps according to size based on initial pathology.

term "serrated polyps" was first used by Longacre and FenoglioPreiser [19]. In 1996, Torlakovic and Snover identified undifferentiated serrated polyps characterized by their large size and proximal location and histologically classified them based on their architectural distortion [20]. Since then, serrated polyps have become a recognized and established separate entity, but an understanding of the different subtypes did not become widespread until approximately a decade ago.

Recent studies have reported prevalence rates for SSA/Ps ranging from $0.6 \%$ to $13.8 \%$ based on detection by colonoscopy [21-23], and most studies have reported rates between $1 \%$ and $5 \%$. However, SSA/Ps are more difficult to detect than traditional adenomatous polyps by endoscopy because they are flat, covered with a mucus cap, and similar in color to the surrounding mucosa. Therefore, the frequency of serrated polyps varies depending on study design and the endoscopist, and consequently, there has 
been a tendency to underreport the number and malignant potential of serrated polyps. The lack of standardization is another reason for the low rate; the diagnosis varies depending on the individual pathologist's definition of SSA/Ps. Unfortunately, other than the report by Lash et al. [21], there have been few reports of large samples of endoscopically resected colorectal polyps.

Lash et al. reported that $1.7 \%$ of a population of 179,111 patients with 290,810 mucosal polyps had SSA/Ps (with and without dysplasia), which were more commonly detected in women and primarily in the right colon. They also reported that $0.9 \%$ (21/2416 lesions) of the patients with SSA/Ps had adenocarcinoma. In the current study, the overall frequency of SSA/Ps was $3.0 \%$ of 21,048 total resected polyps. TSAs are much less common than SSA/Ps, accounting for only $0.6 \%$ of polyps. Compared with the data reported by Lash et al., the frequency of SSA/Ps in our study was slightly higher, but the adenocarcinoma coexistence rate (1.3 $\%)$ was similar. They speculated that the low frequency likely reflected the conservative criteria they used for the inclusion of polyps as SSA/Ps. We hypothesize that the different frequencies of SSA/Ps are due to different diagnostic criteria and treatment strategies used by each endoscopist or pathologist, which have impeded an accurate estimation of the prevalence of SSA/Ps; this remains a major problem regarding SSA/Ps that must be solved in many institutions, including high-volume centers.

Kim et al. recently reported that 21 (18.8\%) of 112 TSAs examined contained an admixture of serrated and traditional adenomatous dysplasia, and 9 (8.0\%) of these TSAs showed intramucosal adenocarcinoma [24]. The frequency of coexisting adenocarcinoma in the TSAs in the present study was lower than that reported by Kim et al. However, they analyzed samples from surgical pathology files, and the polyps were relatively larger $(3-63 \mathrm{~mm})$ than ours; both factors likely contributed to the difference in the coexisting adenocarcinoma incidence in TSAs. In addition, the total number of TSAs evaluated in the present study was relatively small; therefore, we believe further studies are needed to determine the risk of malignancy in TSAs.

We performed ROC analysis to predict risk factors for coexistent carcinoma in serrated polyps. A tumor size of $10 \mathrm{~mm}$ was found to be the optimal cut-off value for coexisting adenocarcinoma. In addition, males accounted for a large proportion of the patients with SSA/Ps (68.6\%), but conversely, females accounted for 80.0 $\%$ of those with coexisting adenocarcinoma. In female patients with serrated polyps larger than $10 \mathrm{~mm}$, the coexisting adenocarcinoma rate was $6.1 \%(\boldsymbol{O}$ Fig. 5 ). Thus, the coexisting adenocarcinoma rate of SSA/Ps in the high-risk group was similar to that among patients with traditional adenomatous polyps. Therefore, endoscopically detected and resected polyps with a serrated phenotype are not always at high risk of becoming malignant. However, the rate of progression from a serrated lesion to CRC is unknown and may depend on MSI-H or BRAF mutation status.

There has been one report of a patient who developed CRC from SSA/Ps in only 8 months [25]. However, another report indicated that it took 15 years for CRC to develop from SSA/Ps [21]. Therefore, it is difficult to predict which serrated polyps have the potential to become malignant. Moreover, Schreiner et al. reported that large serrated polyps were associated with an increased risk for synchronous advanced neoplasia [7]. However, there is insufficient information available regarding the natural history and epidemiology of SSA/Ps and TSAs.

Furthermore, there is no surveillance strategy for serrated lesions after polypectomy. In the current study, the complete en bloc resection rates for SSA/Ps and TSAs were 65.2 and $62.4 \%$, respec-

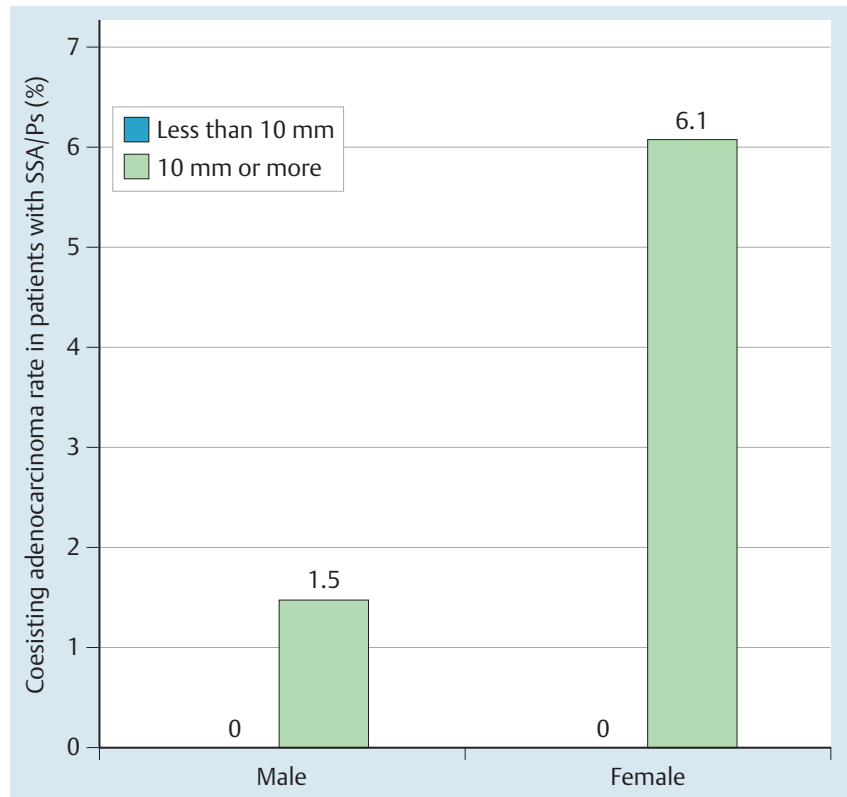

Fig. 5 Incidence of coexisting cancer in patients with at high risk of malignancy.

tively. Pohl et al. reported that the predictors of incomplete resection included the endoscopist, increased polyp size, and SSA/ $P$ rather than traditional adenoma [26]. In this study, the overall rate of complete resection of sessile serrated lesions was $69.0 \%$, which is similar to our data presented here. The rate of incomplete resection was higher for these polyps than for traditional adenomatous polyps (7.2\%) [26]. We experienced one case of local SSA/Ps recurrence due to positive findings at the cut end (data not shown). It is difficult to perform a complete en bloc resection because SSA/Ps are flat, similar in color to the surrounding normal mucosa, and contain unclear edges. Rex et al. recently reported that the rate of recurrence after ER was similar for SSA/Ps and traditional adenomas [27]. These findings suggest that the best surveillance strategy after ER for serrated polyps might be the same as that for traditional adenomatous polyps.

There are several limitations of this retrospective study. First, we did not have strict indications for ER of colorectal polyps. Generally, most colorectal polyps $<5 \mathrm{~mm}$ were not resected in this study; resecting all of the small polyps would have been time consuming, and we previously thought that such small lesions developed slowly and required a long period of time to become invasive cancers. We believed that hyperplastic polyps were harmless, and we thus tended to ignore them, even when they were larger than $5 \mathrm{~mm}$. Unfortunately, it was difficult to stratify the patients based on initial colonoscopy or initial ER, but if we did not achieve a clean colon in the initial colonoscopy, the patients underwent a repeat ER to attain a clean colon, except in cases of small polyps. Therefore, the current findings may not reflect the precise frequency of serrated polyps among patients with colorectal polyps, but the data are valid regarding the frequency of clinically resected colon polyps. Second, there was an endoscopic diagnostic bias in our study. We did not evaluate interobserver variation in assessments of macroscopic appearance, polyp size or SSA/P versus hyperplastic polyp differentiation. Third, there was a pathology diagnostic bias in our study. $\odot$ Supplementary Fig. 2 shows the trends in the percentages of resected hyperplastic polyps and serrated polyps among all resected colo- 
rectal polyps in the current study. We first considered that SSA/Ps diagnosed in the early period may have been misdiagnosed as hyperplastic polyps, but the percentage of hyperplastic polyps remained constant at approximately $5 \%$ during the observation period. In contrast, the percentage of serrated polyps rapidly increased after 2009, but since then, the percentages of hyperplastic polyps and serrated polyps have been similar. A new pathologist (Dr. Adachi) replaced the previous pathologist in 2009, perhaps suggesting that the frequency of polyps diagnosed as SSA/ Ps greatly depends on the pathologist. In addition, our hospital introduced narrow-band imaging endoscopy in 2007; therefore, use of advanced endoscopy and the change in pathologist may have been the main reasons for the higher rate of resection of serrated polyps in the latter period.

In conclusion, the overall frequency of SSA/Ps was 3.0\% among all resected polyps in the present study; the frequency of serrated polyps varied in previous studies depending on study design. TSAs were much less common than SSA/Ps, accounting for only $0.6 \%$ of the polyps in this study. Among the resected colorectal polyps, the rate of coexisting adenocarcinoma in serrated polyps was significantly lower than that in traditional adenomatous polyps, but resection perhaps should be recommended to females with serrated polyps $\geq 10 \mathrm{~mm}$ because of the increased risk of coexistent carcinoma.

\section{Competing interests: None}

Institutions

${ }^{1}$ Department of Gastroenterology, Toyonaka Municipal Hospital, Osaka, Japan

2 Department of Gastroenterology, Japan Community Healthcare

Organization, Osaka Hospital, Osaka, Japan

3 Department of Pathology, Toyonaka Municipal Hospital, Osaka, Japan

\section{References}

1 Jass JR. Serrated route to colorectal cancer: back street or super highway? J Pathol 2001; 193: 283-285

2 Rembacken BJ, Fujii T, Cairns A et al. Flat and depressed colonic neoplasms: a prospective study of 1000 colonoscopies in the UK. Lancet 2000; 355: 1211 - 1214

3 Goldstein NS, Bhanot P, Odish E et al. Hyperplastic-like colon polyps that preceded microsatellite-unstable adenocarcinomas. Am J Clin Pathol 2003; 119: 778 - 796

4 Rex DK, Ahnen DJ, Baron JA et al. Serrated lesions of the colorectum: review and recommendations from an expert panel. Am J Gastroenterol 2012; 107: 1315-1329; quiz 1314, 1330

5 Huang CS, Farraye FA, Yang $S$ et al. The clinical significance of serrated polyps. Am J Gastroenterol 2011; 106: 229-240; quiz 241

6 Snover DC. Update on the serrated pathway to colorectal carcinoma. Hum Pathol 2011; 42: 1 - 10

7 Schreiner MA, Weiss DG, Lieberman DA. Proximal and large hyperplastic and nondysplastic serrated polyps detected by colonoscopy are associated with neoplasia. Gastroenterology 2010; 139: 1497-1502
8 Hiraoka S, Kato J, Fujiki $S$ et al. The presence of large serrated polyps increases risk for colorectal cancer. Gastroenterology 2010; 139: $1503-1510,1510$ e 1501 - e1503

9 Snover DC, Jass JR, Fenoglio-Preiser C et al. Serrated polyps of the large intestine: a morphologic and molecular review of an evolving concept. Am J Clin Pathol 2005; 124: 380-391

10 Mattar W, Rex DK. Large sessile adenomas are associated with a high prevalence of synchronous advanced adenomas. Clin Gastroenterol Hepatol 2008; 6: 877-879

11 Salama M, Ormonde D, Quach T et al. Outcomes of endoscopic resection of large colorectal neoplasms: an Australian experience. J Gastroenterol Hepatol 2010; 25: 84-89

12 Inoue H, Kashida H, Kudo $S$ et al. The Paris endoscopic classification of superficial neoplastic lesions: esophagus, stomach, and colon: November 30 to December 1, 2002. Gastrointest Endosc 2003; 58: 3-43

13 Fujimori Y, Fujimori T, Imura J et al. An assessment of the diagnostic criteria for sessile serrated adenoma/polyps: SSA/Ps using image processing software analysis for Ki67 immunohistochemistry. Diagn Pathol 2012; 7: 59

14 Lane $N$. The precursor tissue of ordinary large bowel cancer. Cancer Res 1976; 36: 2669-2672

15 Clark JC, Collan Y, Eide TJ et al. Prevalence of polyps in an autopsy series from areas with varying incidence of large-bowel cancer. Int J Cancer 1985; 36: 179-186

16 Correa P, Strong JP, Reif A et al. The epidemiology of colorectal polyps: prevalence in New Orleans and international comparisons. Cancer 1977; 39: $2258-2264$

17 Williams AR, Balasooriya BA, Day DW. Polyps and cancer of the large bowel: a necropsy study in Liverpool. Gut 1982; 23: $835-842$

18 Vatn $M H$, Stalsberg $H$. The prevalence of polyps of the large intestine in Oslo: an autopsy study. Cancer 1982; 49: 819-825

19 Longacre TA, Fenoglio-Preiser CM. Mixed hyperplastic adenomatous polyps/serrated adenomas. A distinct form of colorectal neoplasia. Am J Surg Pathol 1990; 14: 524-537

20 Torlakovic E, Snover DC. Serrated adenomatous polyposis in humans. Gastroenterology 1996; 110: 748-755

21 Lash RH, Genta RM, Schuler CM. Sessile serrated adenomas: prevalence of dysplasia and carcinoma in 2139 patients. J Clin Pathol 2010; 63: $681-686$

22 Spring KJ, Zhao ZZ, Karamatic R et al. High prevalence of sessile serrated adenomas with BRAF mutations: a prospective study of patients undergoing colonoscopy. Gastroenterology 2006; 131: 1400-1407

23 Carr NJ, Mahajan H, Tan KL et al. Serrated and non-serrated polyps of the colorectum: their prevalence in an unselected case series and correlation of BRAF mutation analysis with the diagnosis of sessile serrated adenoma. J Clin Pathol 2009; 62: 516-518

$24 \mathrm{Kim}$ KM, Lee EJ, Kim YH et al. KRAS mutations in traditional serrated adenomas from Korea herald an aggressive phenotype. Am J Surg Pathol 2010; 34: 667-675

25 Oono Y, Fu K, Nakamura H et al. Progression of a sessile serrated adenoma to an early invasive cancer within 8 months. Dig Dis Sci 2009; 54: 906-909

26 Pohl H, Srivastava A, Bensen SP et al. Incomplete polyp resection during colonoscopy-results of the complete adenoma resection (CARE) study. Gastroenterology 2013; 144: 74-80 e71

27 Rex KD, Vemulapalli KC, Rex DK. Recurrence rates after EMR of large sessile serrated polyps. Gastrointest Endosc 2015; 8: 538-541 
Supplementary Table 1 Patient details and characteristics of serrated polyps with adenocarcinoma

\begin{tabular}{|c|c|c|c|c|c|c|c|}
\hline Case & Sex & Age & Location & Tumor size (mm) & Macroscopic type & Histologic depth & SSA/P or TSA derived \\
\hline a & $\mathrm{F}$ & 55 & $\mathrm{R}$ & 10 & Isp & M & SSA $/ \mathrm{P}$ \\
\hline b & $\mathrm{F}$ & 87 & A & 12 & Isp & M & TSA \\
\hline c & $\mathrm{F}$ & 48 & $S$ & 12 & Isp & $M$ & SSA/P \\
\hline d & $\mathrm{F}$ & 66 & A & 12 & Ila & $\mathrm{SM}, 1000 \mu \mathrm{m}$ & SSA $/ P$ \\
\hline e & M & 65 & C & 15 & Isp & M & SSA/P \\
\hline$f$ & M & 57 & S & 15 & Ip & $\mathrm{SM}, 2000 \mu \mathrm{m}$ & SSA/P \\
\hline g & $\mathrm{F}$ & 62 & S & 20 & Isp & M & SSA/P \\
\hline $\mathrm{h}$ & $\mathrm{F}$ & 67 & A & 20 & Ila & M & SSA /P \\
\hline i & $\mathrm{F}$ & 75 & T & 30 & Ila & $M$ & SSA/P \\
\hline
\end{tabular}

F: female; M (sex): male; C: cecum; A: ascending colon; T: transverse colon; S: sigmoid colon; R: rectum; M (histological depth): mucosa; SM: submucosa; TSA: traditional serrated adenoma; SSA/P: sessile serrated adenoma/polyp
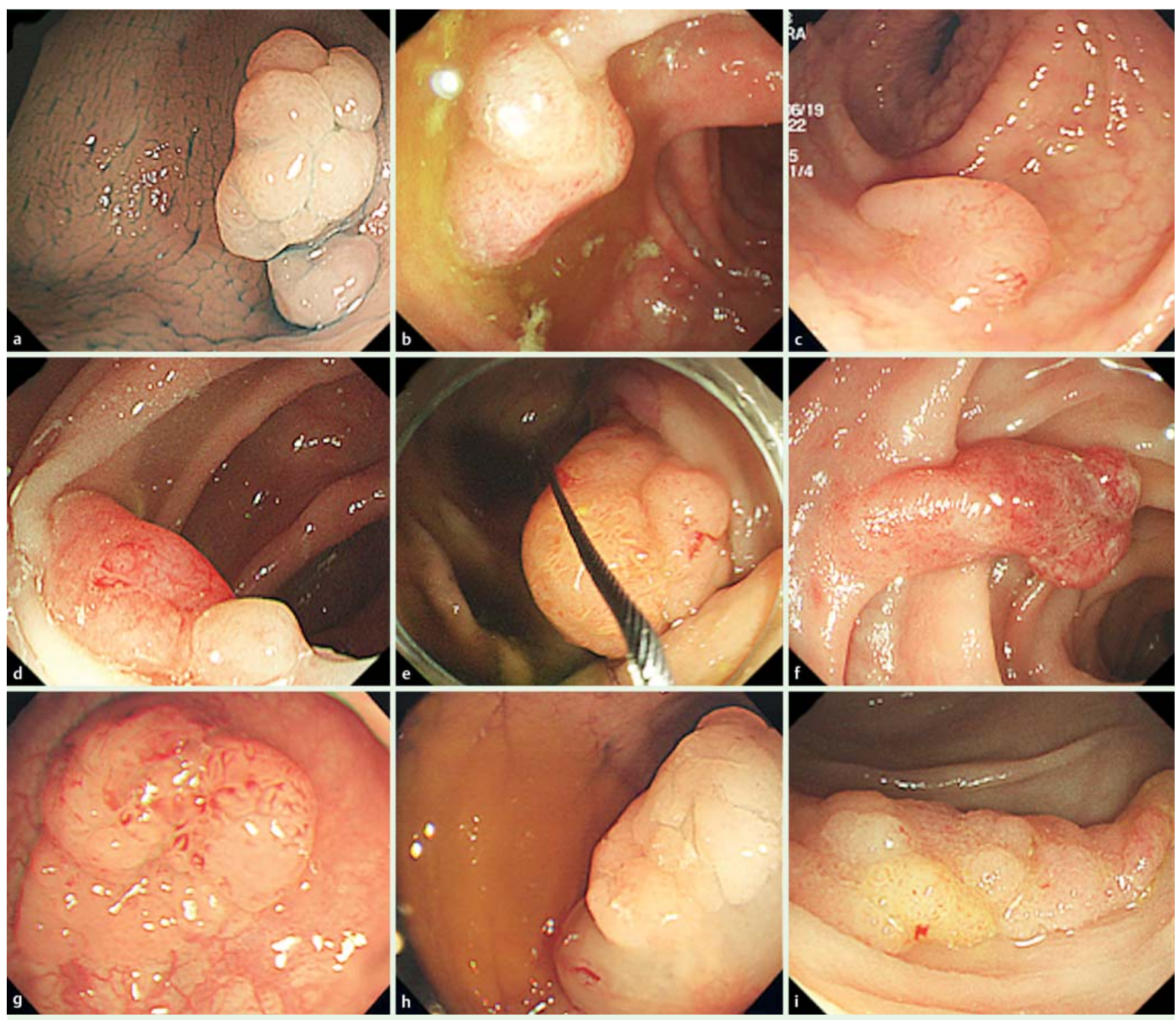

Supplementary Fig. 1 Endoscopic images of patients with serrated polyps with adenocarcinoma (Cases a-i, Supplementary Table 1, h: after injection of saline into the submucosa). 


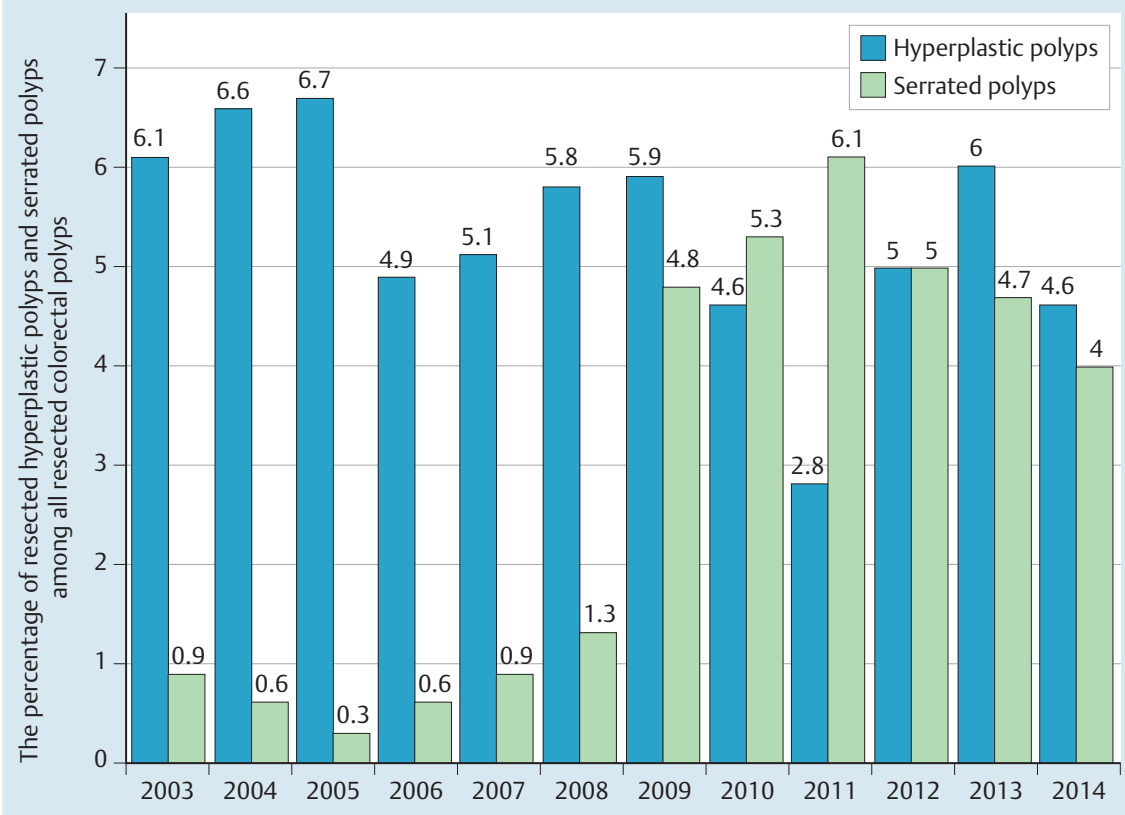

Supplementary Fig. 2 Trends in percentages of resected hyperplastic polyps and serrated polyps among all resected colorectal polyps in the current study. 